\title{
Dynamic Tunneling Junctions at the Atomic Intersection of Two Twisted Graphene Edges
}

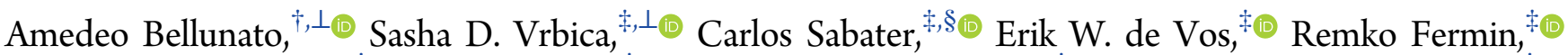 \\ Kirsten N. Kanneworff, ${ }^{\ddagger}$ Federica Galli, ${ }^{\ddagger}$ Jan M. van Ruitenbeek, ${ }^{*}{ }^{\ddagger}$ a and Grégory F. Schneider ${ }^{* \dagger}$ \\ ${ }^{\dagger}$ Faculty of Science, Leiden Institute of Chemistry, Leiden University, Einsteinweg 55, 2333CC Leiden, The Netherlands \\ ${ }^{\ddagger}$ Faculty of Science, Huygens-Kamerlingh Onnes Laboratory, Leiden University, Niels Bohrweg 2, 2333CA Leiden, The Netherlands
}

\section{Supporting Information}

ABSTRACT: The investigation of the transport properties of single molecules by flowing tunneling currents across extremely narrow gaps is relevant for challenges as diverse as the development of molecular electronics and sequencing of DNA. The achievement of well-defined electrode architectures remains a technical challenge, especially due to the necessity of high precision fabrication processes and the chemical instability of most bulk metals. Here, we illustrate a continuously adjustable tunneling junction between the edges of two twisted graphene sheets. The unique property of the graphene electrodes is that the sheets are rigidly supported all the way to the atomic edge. By analyzing the tunneling current characteristics, we also demonstrate that the spacing across the gap junction can

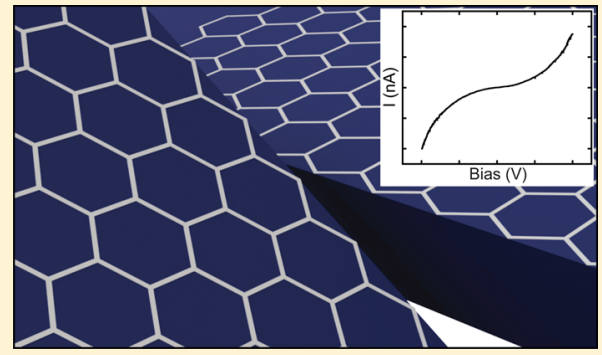
be controllably adjusted. Finally, we demonstrate the transition from the tunneling regime to contact and the formation of an atomic-sized junction between the two edges of graphene.

KEYWORDS: Graphene, graphene edges, nanogap, tunneling junction, molecular break junction, molecular electronics

$\mathrm{T}$ he great potential that graphene offers as an electrode material for addressing individual molecules has been widely recognized. This is of particular importance in the study of electron transport across individual molecules, ${ }^{1-3}$ in the development of molecular electronics, ${ }^{4,5}$ and for direct electron current readout in the quest of sequencing biopolymers. ${ }^{6,7}$ In a typical essay in any of these fields of research, a voltage is applied across a nanoscale gap between two metallic electrodes where the measured current contains information on the nature of the molecule bridging this gap. In absence of molecules in the gap, the current that flows is a pure tunneling current, resulting from the finite overlap of the exponentially decaying electron wave functions on either side of the gap. Currently, nearly all experiments have been performed using some form of break junction devices with metallic electrodes, mostly gold. ${ }^{8}$ Metallic electrodes pose serious limitations, associated with poor characterization and poor reproducibility of the molecule-electrode bonding configurations. The size and shapes of the metal electrodes are generally unknown, the shape and surface coverage are subject to rapid chemical and geometrical modifications, and the radius of curvature of the electrodes is much larger than the size of the molecules under study, notably in the case of the widely used gold electrodes at room temperature. ${ }^{9}$ The large size and the poorly known shape of the electrodes limits accurate comparison with computational modeling. ${ }^{10}$ Moreover, (gold) metal electrodes offer a wide variety of choice for the position of the molecules between the electrodes and for the bonding motifs, which lead to more unknowns in the analysis of the observations. ${ }^{11}$ The size of the electrodes is of particular interest in developing direct current readout for sequencing of biopolymers, in which case the extent of the electrodes in the direction along the length of the biopolymer should ideally be smaller than the size of the individual building blocks forming the biopolymer.

For many of those concerns, the use of graphene edge electrodes offers a promising approach. Graphene is a good conductor of electricity and the size of the layer in the direction perpendicular to its plane is given by the size of just a single carbon atom. Further benefits of the use of graphene include the stability of the covalently bonded lattice, the fact that image charges are nearly absent, which greatly simplifies the comparison with computational models, the fact that the edges offer a limited range of bonding motifs, which can be further exploited by edge-specific chemical decorations. Graphene electrodes can be contacted via $\pi$-stacking ${ }^{3,12}$ or through covalent bonds, ${ }^{13-15}$ introducing selective docking sites for molecular trapping and characterization. ${ }^{16,17}$

Several approaches toward exploiting graphene electrodes for addressing individual molecules have already been reported, where the challenge is the required small size of the gap between the electrodes. By exploiting the surface tension of an evaporating solvent a freely suspended sheet of graphene can be torn into forming a tunneling junction on a $\mathrm{Si} / \mathrm{SiO}_{2}$ substrate. ${ }^{18}$ Alternatively, feedback-controlled electroburning can be used for fabricating graphene nanogaps, ${ }^{1-4,19}$ or high-resolution

\footnotetext{
Received: January 12, 2018

Revised: February 23, 2018

Published: March 7, 2018
} 
electron-beam patterning in combination with oxygen plasma etching. ${ }^{20}$ These methods have in common that the size of the gap cannot be precisely and freely designed, and the resulting junctions are static: once created, the size of the nanogap cannot be adjusted. A drawback in the context of applications for sequencing is the fact that both the electrodes are sculpted on a common substrate that covers the gap between the electrodes, physically preventing the flow of molecules across the gap.

In this paper, we describe the fabrication and characterization of two twisted graphene edge electrodes supported to the atomic edge, where electrons tunnel between the two carbon atoms facing each other. Using piezoelectric actuators of a modified scanning tunneling device the two graphene edges are positioned relative to each other with subatomic precision, leaving an empty gap between the two facing carbon atoms that could be eventually used for the translocation of molecules (Figure 1).

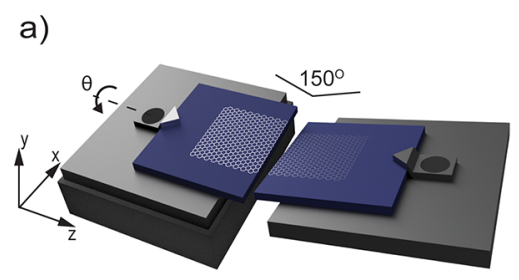

b)
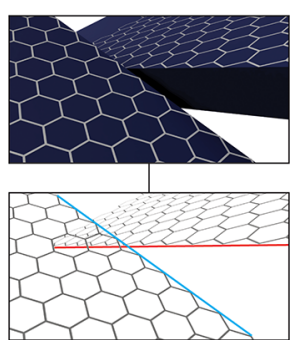

Figure 1. Schematic illustration of the method for producing a dynamically adjustable graphene-graphene junction. (a) Two independent graphene electrodes, each supported to the atomic edge, can be approached edge-to-edge with subatomic precision, creating a junction between two single rows of carbon edge atoms. The electrodes are twisted and tilted in order to form a well-defined intersection point between the two electrodes and for preventing mechanical contact of the supports. The twist angle $\theta$ is between 10 and $45^{\circ}$. (b) Illustration of the carbon-carbon point contact formed at the intersection of two graphene sheet edges.

We prepared two independent supports with atomically sharp edges by cleaving-off a polished $\mathrm{Si} / \mathrm{SiO}_{2}$ wafer after introducing a notch on the surface at the edge of the wafer with a diamond knife. The notch initiates a crack that develops along a high-symmetry crystallographic direction of the silicon, yielding straight edge profiles. The supports are mounted over a slit in a holder, facing each other at a distance of about a millimeter (Figure 2a). A graphene sheet supported by a layer of polycarbonate $^{21}$ (PCA) is deposited on top of the two wafer halves, bridging the gap between them (Figure 2b). The graphene is obtained by chemical vapor deposition (CVD) on a copper film, followed by spin coating PCA to cover the graphene. The copper is etched in $0.5 \mathrm{M}$ ammonium persulfate, while the polymer works as support. The film is rinsed three times with ultrapure water to remove residuals of ammonium persulfate and transferred over the wafers. Subsequently, the holder with the wafers and the polymer-supported graphene are exposed from below to an isotropic $\mathrm{H}_{2}$ plasma. The plasma removes the graphene suspended over the slit, while the polymer protects the parts that cover the $\mathrm{SiO}_{2}$ supports.

Next, the PCA coating is dissolved in chloroform, the assembly is rinsed in methanol and isopropanol, and the resulting graphene edge electrodes are imaged using optical microscopy (Figure $2 \mathrm{c}, \mathrm{d}$ ). The protective role of the polymer a)

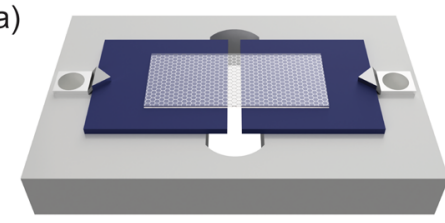

c)

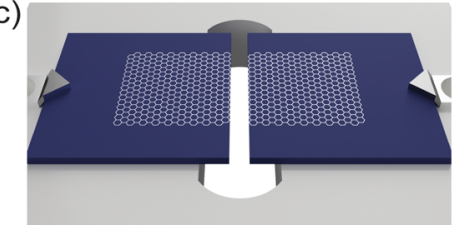

b)

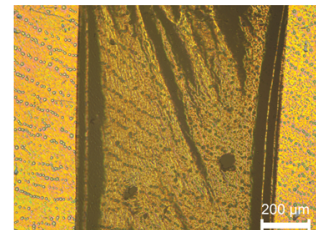

d)

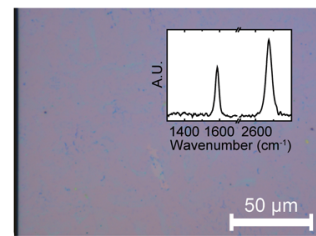

Figure 2. Fabrication of graphene edge electrodes. (a) Schematic illustration of a graphene layer protected by a polymer coating (white shading covering the graphene) bridging the gap between two $\mathrm{Si} / \mathrm{SiO}_{2}$ wafer halves mounted over the slit of a holder. (b) Optical microscopy image showing the two $\mathrm{Si} / \mathrm{SiO}_{2}$ wafer halves (yellow) bridged by the freestanding polymer-coated graphene (dark yellow). (c) Schematic illustration of the setup after plasma etching and polymer removal. (d) Optical microscopy image of the supported graphene edge (visible at the far left) after polymer removal. The inset shows a Raman spectrum of the graphene after plasma etching and polymer removal.

toward the highly reactive hydrogen plasma is confirmed by the presence of a strong $G$ peak ${ }^{22}$ around $1590 \mathrm{~cm}^{-1}$ and a negligible $\mathrm{D}$ peak around $1340 \mathrm{~cm}^{-1}$.

Prior to performing tunneling measurements, we characterized the graphene edge electrodes using Raman spectroscopy, scanning electron microscopy (SEM), and atomic force microscopy (AFM), see Figure 3. The mapping in Figure 3a overlaps Raman peak intensity distributions over the surface of the electrode near the edge with the $2 \mathrm{D}$ band around 2700 $\mathrm{cm}^{-1}$ shown in green, the $\mathrm{G}$ band at $1590 \mathrm{~cm}^{-1}$ shown in blue, and the $\mathrm{D}$ band at $1340 \mathrm{~cm}^{-1}$ shown in red. The graphene extends uniformly all over the surface of the $\mathrm{SiO}_{2}$ substrate, a)

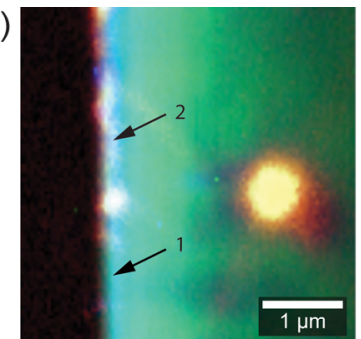

C)

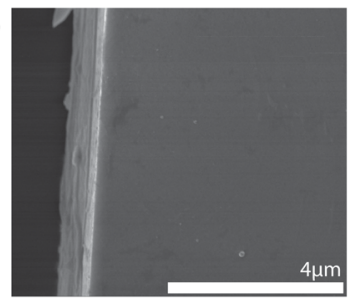

b)

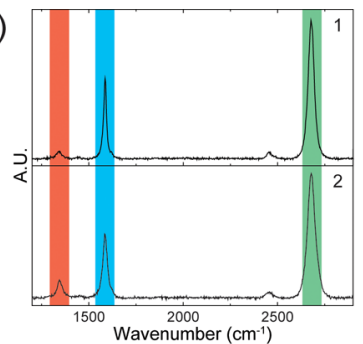

d)

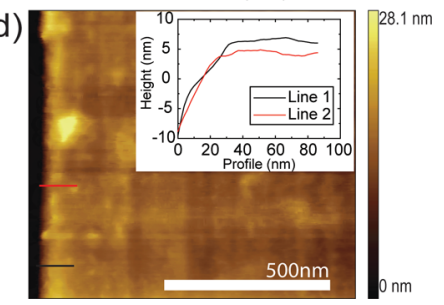

Figure 3. Characterization of the graphene edge. (a) Overlay of the Raman mapping of the $\mathrm{D}$ band $\left(1340 \mathrm{~cm}^{-1}\right.$, red tone), G band (1590 $\mathrm{cm}^{-1}$, blue tone) and $2 \mathrm{D}$ band $\left(2667 \mathrm{~cm}^{-1}\right.$, green tone). The large yellow island is due to a polymer residue. (b) Raman spectra of two single points on the edge, as marked in panel a. (c) SEM micrograph of the edge electrode. (d) AFM topography of a graphene edge electrode. Inset: AFM height profiles of the graphene extending to the edge of the support. 

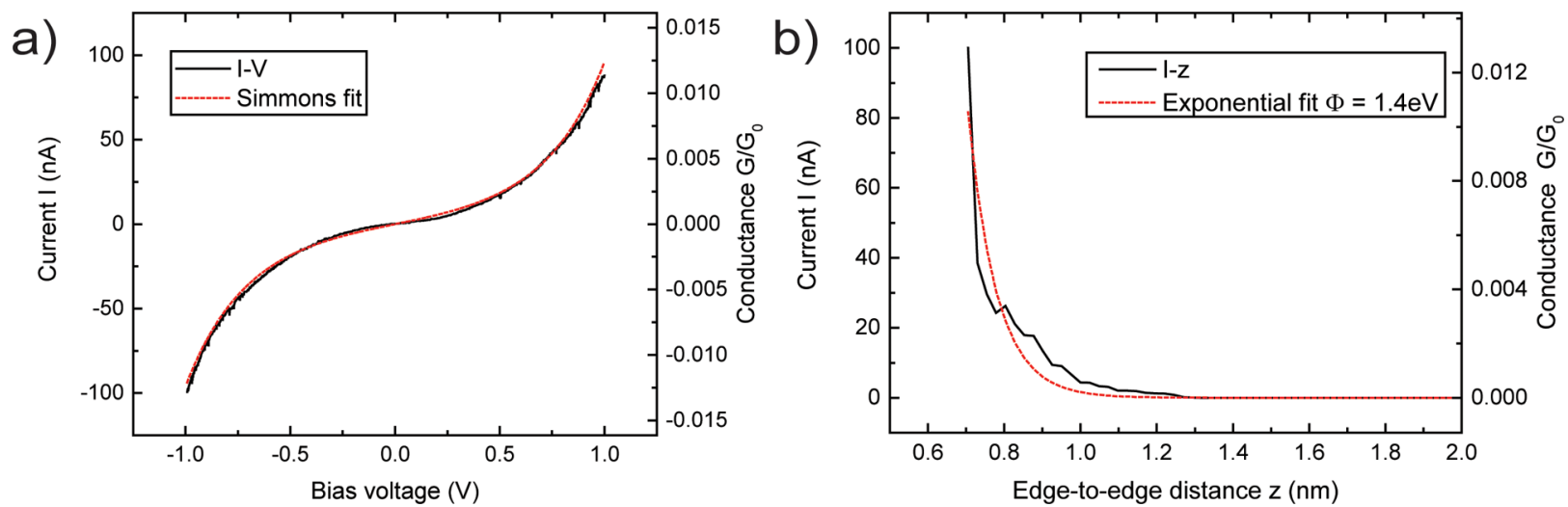

Figure 4. Electrical characterization of a dynamically adjustable graphene-graphene edge tunneling junction under ambient conditions. (a) Black curve, current-voltage characteristics of the junction in the tunneling regime. Red curve, fit to the Simmons model with a vacuum gap size of $1.3 \mathrm{~nm}$, barrier height of $1.4 \mathrm{eV}$. (b) Current-distance, $I-z$, characteristics of the tunnel junction (black line). Red curve, exponential fit for tunneling against gap size, adopting the barrier height obtained from the fit in (a). The left axis in (a,b) shows the measured current, while the right axis shows the conductance proportional to the quantum of conductance $\mathrm{G}_{0}$ approximated to $77.5 \mu \mathrm{S}$.

beyond which the mapping appears black (left side of the image). The uniformity of the color scale illustrates the quality of the graphene, and representative single-spot spectra acquired at the edge of the support are shown in Figure $3 \mathrm{~b}$. The ratio of the intensities of the $2 \mathrm{D}$ and the $\mathrm{G}$ peaks is in agreement with the ratio expected for monolayer graphene, while the onset of a $\mathrm{D}$ peak at the edge is characteristic of the breaking in the symmetry of the graphene lattice. ${ }^{23}$ The relative intensity of the $\mathrm{D}$ band with respect to the $\mathrm{G}$ and $2 \mathrm{D}$ bands is an indicator of the uniformity of the graphene lattice. ${ }^{24}$ The bright yellow spot at the right side of the image is attributed to a polymer residue. Such polymer residues could be removed by high-temperature annealing. However, the high temperatures required for cleaning the surface may induce ruptures of the graphene sheet, therefore displacing the graphene from the edge of the substrate due to differences in thermal expansion coefficients of graphene and $\mathrm{Si} / \mathrm{SiO}_{2}$. Accordingly, we avoid annealing and accept the presence of some polymer residues.

Figure 3c shows a SEM micrograph of a graphene edge electrode on $\mathrm{Si} / \mathrm{SiO}_{2}$ support. The small darker regions indicate the formation of local graphene bilayer islands during the CVD growth. ${ }^{25}$ Additionally, small white features are attributed to polymer residues. We observe no variations in the color contrast of the graphene film on top of the $\mathrm{SiO}_{2}$ in proximity of the edge, suggesting the continuous extension of the film.

Neither SEM nor Raman can resolve the extension of the graphene sheet to the edge of its substrate down to the nanometer scale. We refine the characterization toward higher resolution by performing atomic force microscopy (AFM). Special care was taken in choosing the appropriate scanning parameters in order to be able to image at the sharp edge. The line-scan direction was chosen perpendicular to the edge of the wafer and the feedback was set to a low value in order to prevent fast descent of the cantilever once beyond the edge and subsequent crashing. Figure $3 \mathrm{~d}$ shows a tapping mode AFM topography image of the surface of graphene up to the edge and beyond the wafer support (black area on the left in Figure 3d). The bright areas correspond to higher regions such as graphene bilayer areas typical of CVD grown graphene, as well as polymer residues accumulated near the edges of the graphene film during the removal of the polymer. We observe uniformity of the color contrast up to the edge of the wafer within the
AFM resolution $(\sim 5 \mathrm{~nm})$. The inset in Figure $3 \mathrm{~d}$ shows two height profiles perpendicular to the edge electrode.

The final test of whether graphene extends to the edge is in the observation of a tunneling current. Tunneling currents decay exponentially with the distance between the electrodes. If the graphene would be displaced from the edge of the support by more than a few nanometers, no tunneling current could be measured. Tunnel junctions between two graphene edges were formed by approaching a pair of edge electrodes using a modified piezo actuator of a scanning tunneling microscope (STM), operating under ambient conditions. The piezo actuator permits the controlled approach into tunneling distance of the graphene layers on the $\mathrm{Si}$ supports. The wafers are tilted downward forming an angle of $15^{\circ}$ in order to avoid the Si substrates hampering the approach of the electrodes. A twist of one of the supports around the $Z$-axis (see Figure 1 ) leads to the formation of a single point of intersection between the two graphene edges. Ideally, two single carbon atoms meet at the intersection, constituting an atomic tunneling junction. Piezo actuator controlled displacements in $X$ - and $Y$-directions permit selecting fresh spots for tunneling.

Figure 4a shows an example of the measured current-voltage ( $I-V)$ dependence of a graphene-graphene tunnel junction for a bias voltage sweep of $\pm 1.0 \mathrm{~V}$. The sigmoidal shape of the curve is a distinctive feature of electron tunneling through a potential barrier. Here, the barrier height is determined by the work function, and the width of the barrier is given by the distance between the graphene edges. We employ the Simmons model for tunneling through symmetric barriers ${ }^{26}$ to fit the size of the vacuum gap and the height of the work function (see Supporting Information). From this fit, we obtain a distance of $1.3 \mathrm{~nm}( \pm 5 \%)$ and an effective work function $\Phi=1.4 \mathrm{eV}$ $( \pm 8 \%)$. The work function is significantly lower than the values reported from Kelvin probe microscopy on the face of graphene sheets, which are in the range from 4.45 to $4.8 \mathrm{eV}$, depending on the doping. ${ }^{27}$ Much lower work functions, ranging from 0.25 to $1.0 \mathrm{eV}$ are typically found from Simmons fits and edge tunneling, as reported for graphene nanogaps obtained from electroburning. ${ }^{2,3,5}$ In our case, the chemistry of the edges under fabrication conditions influences the work function. ${ }^{28-30}$ Because we etch the sample in $\mathrm{H}_{2}$ plasma, we assume most of the carbon dangling bonds to be hydrogenated, which reduces the work function below $4 \mathrm{eV} .^{31}$ The presence of chemisorbed 
a)

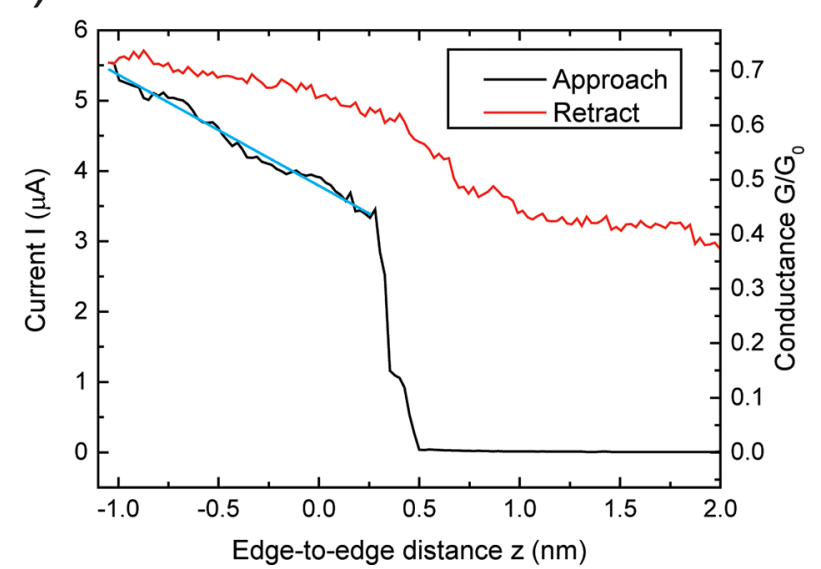

b)

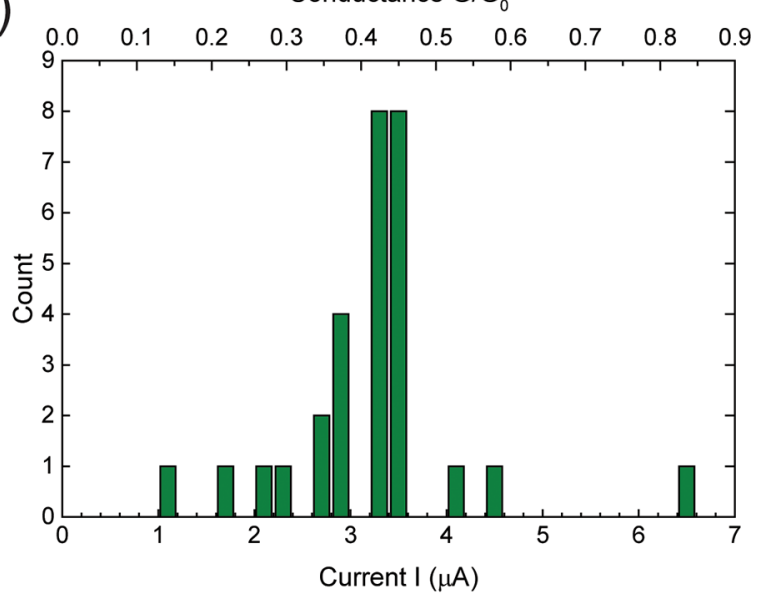

Figure 5. From tunneling regime to point contact. (a) Black, approach curve obtained for the "closing-up" of the nanogap, showing a transition from the exponential tunneling regime to a linear point contact regime (indicated by the blue line fit) at a bias voltage of $0.1 \mathrm{~V}$. Red, retraction curve showing hysteresis that we attribute to bond formation. (b) Green bars, histogram of the point contact formation (current at the kink in (a)) for 29 junctions at a bias voltage of $0.1 \mathrm{~V}$. The left and bottom scales in (a,b), respectively, show the measured current, while the scales at the opposite sides are converted to conductance in units of the quantum of conductance $G_{0}$. The measurements are performed under ambient conditions.

and physisorbed species at the edges of the electrodes under ambient conditions is expected to further reduce the work function. ${ }^{3}$

The independent positioning of our edge electrodes allows fine adjustment of the gap. Figure $4 \mathrm{~b}$ shows a current-distance curve, $I-z$, measured at a bias voltage of $\mathrm{V}=0.1 \mathrm{~V}$, black curve. We fit the measured $I-z$ curve with an exponential function (in red) using the effective work function $\Phi$ as obtained from the $I-V$ characteristic in Figure 4a. Because the shape of the curve is fully determined and only the exponential prefactor is freely adjustable, the fit provides strong confirmation of the vacuum tunneling origin of the current. We are not aware of any previous methods for recording the tunneling current between the edges of two graphene sheets as a function of their distance. Although the measured curve still has some irregularities due to vibrations and possibly due to fluctuations in adsorbents, the observed exponential dependence confirms that we are able to tune the gap of a tunnel junction with subnanometric precision.

Moving the graphene electrodes closer together the electron transport across the junction transforms from the tunneling to the contact regime. The twisted configuration should lead to an initial point contact between single carbon atoms at the intersection of the two edges of the graphene electrodes. Figure 5 a shows an $I-z$ curve for a junction across the transition from the tunnel regime to the point contact regime, at a bias voltage of $0.1 \mathrm{~V}$, black curve. Following the exponential increase of the current in tunnel regime, we observe a kink at about $3 \mu \mathrm{A}$, indicating a switch to contact. Pushing the electrodes further into contact yields the current to increase further, approximately linearly. The onset of the linear regime is found at a contact resistance of around $30 \mathrm{k} \Omega$, which is of the order of the quantum of resistance expected for point contacts, $R_{q}=12.9$ $\mathrm{k} \Omega$, equivalent to a quantum of conductance $G_{0}=77.5 \mu \mathrm{S}$. Figure $5 \mathrm{~b}$ shows a histogram of the current values observed at the kink for 29 independent junctions formed. The histogram shows that statistically the onset of the linear resistance regime is found at $28 \mathrm{k} \Omega$ or $0.46 \mathrm{G}_{0}$. Note that this resistance is the actual contact quantum resistance, measured in series with the resistance of the graphene electrodes (see Supporting Information). The estimated quantum point contact resistance is $11 \pm 3 \mathrm{k} \Omega$ or $1.2 \pm 0.3 \mathrm{G}_{0}$, which is in good agreement with the value calculated for carbon-carbon atomic contacts at low strain, ${ }^{32}$ and with the quantum of resistance.

The linear trend to higher conductance after the kink suggests that the contact between the edges of graphene of the two graphene sheets can be increased by indentation. Note that we are only indenting the electrodes by a few atomic distances. The linear trend in conductance agrees with the twodimensional geometry of the graphene sheets and differs from the nearly quadratic growth of conductance for threedimensional metallic contacts.

When we retract the electrodes after this indentation in order to restore the vacuum gap, we observe hysteresis, as shown by the red curve in Figure 5a. During retraction, the conductance remains high and after a small downward step near the point where the initial contact was formed, the contact is not broken even for further stretching of the junction over several nanometers. The persistence of high conductance suggests that strong bonds have been formed under the influence of the high mechanical pressure, possibly in combination with the potential of $0.1 \mathrm{~V}$ applied across the junction. We speculate that carbon-carbon bonds have been formed between the graphene sheets during indentation although all carbon bonds initially should be saturated. The long distance over which a highconductance state survives (up to $3 \mathrm{~nm}$ or more) suggests that elongated atomic-chain like structures are formed in the retraction process, as has been observed previously in transmission electron microscope experiments, ${ }^{32-34}$ and in first-principles molecular dynamics. ${ }^{35-37}$ Accordingly, we observed that after several hundred cycles of point contact formation and retraction at different spots along the edge, the damage to the electrodes hindered reaching the point contact regime and at times even obstructed an exponential increase of the current. Nonetheless, the validity of this speculation will need further experiments for detailed evaluation.

To conclude, we have presented a system consisting of dynamically adjustable graphene-graphene edge tunneling junctions. The independently supported graphene electrodes uniquely allow the fine adjustment of the gap size. The tuneability of the gap is relevant for applications of such 
junctions, especially in the fields of single-molecule characterization, biosensing, and DNA sequencing. The junction can be adapted for sensing in liquid environments, required for the translocation of molecules such as DNA strands across the gap and recording their electric fingerprint. ${ }^{38}$ In testing of the junctions, we monitored the transition from the tunnel regime to atomic-size contact and observed a preferred initial contact resistance value of $28 \mathrm{k} \Omega\left(0.46 \mathrm{G}_{0}\right)$. The presence of hysteresis in the contact formation-and-breaking cycle suggests that bonds are formed between the graphene electrodes under influence of mechanical pressure. With further developments of our system under controlled atmosphere, in liquid environments and at cryogenic temperatures, we believe that our findings will advance molecular electronics research, will open the way to research of atomic-size junctions in graphene, and will find applications in sensing and biopolymer sequencing.

\section{ASSOCIATED CONTENT}

\section{S Supporting Information}

The Supporting Information is available free of charge on the ACS Publications website at DOI: 10.1021/acs.nanolett.8b00171.

Simmons model for symmetric barrier; graphene-gold tunneling junction; preparation of the supports; graphene film; tunnelling junction controller; sheet resistance and point contact resistance; Raman characterization; additional figures and references (PDF)

\section{AUTHOR INFORMATION}

\section{Corresponding Authors}

*E-mail: g.f.schneider@chem.leidenuniv.nl.

*E-mail: ruitenbeek@physics.leidenuniv.nl.

\section{ORCID}

Amedeo Bellunato: 0000-0001-7016-4738

Sasha D. Vrbica: 0000-0003-2189-849X

Carlos Sabater: 0000-0001-8586-9976

Erik W. de Vos: 0000-0002-9363-1447

Remko Fermin: 0000-0002-0165-4263

Kirsten N. Kanneworff: 0000-0002-4777-8966

Federica Galli: 0000-0002-1098-8150

Jan M. van Ruitenbeek: 0000-0003-0381-0132

\section{Present Address}

$\$$ (C.S.) Weizmann Institute of Science, Chemical Physics Department, Herzl St 234, 76100, Rehovot, Israel.

\section{Author Contributions}

${ }^{\perp}$ A.B. and S.D.V. contributed equally. The manuscript was written through contributions of all authors. All authors have given approval to the final version of the manuscript.

\section{Funding}

European Union's Seventh Framework Program (FP/20072013)/ERC Grant Agreement 335879 project acronym "Biographene". Netherland's Organization for Scientific Research NWO Grant 12QIM05.

\section{Notes}

The authors declare no competing financial interest.

\section{ACKNOWLEDGMENTS}

We would like to acknowledge the European Research Council under the European Union's Seventh Framework Program (FP/2007-2013)/ERC Grant Agreement 335879 project acronym "Biographene". This work is part of the research program of the Netherland's Organization for Scientific Research, NWO.

\section{REFERENCES}

(1) Prins, F.; Barreiro, A.; Ruitenberg, J. W.; Seldenthuis, J. S.; AliagaAlcalde, N.; Vandersypen, L. M. K.; van der Zant, H. S. J. Nano Lett. 2011, 11, 4607-4611.

(2) Nef, C.; Pósa, L.; Makk, P.; Fu, W.; Halbritter, A.; Schönenberger, C.; Calame, M. Nanoscale 2014, 6, 7249-7254.

(3) Ullmann, K.; Coto, P. B.; Leitherer, S.; Molina-Ontoria, A.; Martín, N.; Thoss, M.; Weber, H. B. Nano Lett. 2015, 15, 3512-3518.

(4) Wen, H.; Li, W.; Chen, J.; He, G.; Li, L.; Olson, M. A.; Sue, A. C.H.; Stoddart, J. F.; Guo, X. Sci. Adv. 2016, 2, e1601113-e1601113.

(5) Lau, C. S.; Mol, J. A.; Warner, J. H.; Briggs, G. A. D. Phys. Chem. Chem. Phys. 2014, 16, 20398-20401.

(6) Lagerqvist, J.; Zwolak, M.; Di Ventra, M. Nano Lett. 2006, 6, 779-782.

(7) Arjmandi-Tash, H.; Belyaeva, L. A.; Schneider, G. F. Chem. Soc. Rev. 2016, 45, 476-493.

(8) Cuevas, J. C.; Scheer, E. Molecular Electronics; World Scientific, 2010.

(9) Agrait, N.; Yeyati, L.; van Ruitenbeek, J. M. Phys. Rep. 2003, 377, 81-279.

(10) Perrin, M. L.; Verzijl, C. J. O.; Martin, C. A.; Shaikh, A. J.; Eelkema, R.; Van Esch, J. H.; Van Ruitenbeek, J. M.; Thijssen, J. M.; Van Der Zant, H. S. J.; Dulić, D. Nat. Nanotechnol. 2013, 8, 282-287.

(11) Paulsson, M.; Krag, C.; Frederiksen, T.; Brandbyge, M. Nano Lett. 2009, 9, 117-121.

(12) Fu, W.; Nef, C.; Tarasov, A.; Wipf, M.; Stoop, R.; Knopfmacher, O.; Weiss, M.; Calame, M.; Schönenberger, C. Nanoscale 2013, 5, 12104.

(13) Baraket, M.; Stine, R.; Lee, W. K.; Robinson, J. T.; Tamanaha, C. R.; Sheehan, P. E.; Walton, S. G. Appl. Phys. Lett. 2012, 100, 233123.

(14) Chua, C. K.; Pumera, M. Chem. Soc. Rev. 2013, 42, 3222.

(15) Liu, Y.; Dong, X.; Chen, P. Chem. Soc. Rev. 2012, 41, 22832307.

(16) Zhang, B.; Cui, T. Appl. Phys. Lett. 2011, 98, 073116.

(17) Xu, G.; Abbott, J.; Qin, L.; Yeung, K. Y. M.; Song, Y.; Yoon, H.; Kong, J.; Ham, D. Nat. Commun. 2014, 5, 4866.

(18) Wang, H. M.; Zheng, Z.; Wang, Y. Y.; Qiu, J. J.; Guo, Z. B.; Shen, Z. X.; Yu, T. Appl. Phys. Lett. 2010, 96, 023106.

(19) Sadeghi, H.; Mol, J.; Lau, C.; Briggs, A.; Warner, J.; Lambert, C. J. Proc. Natl. Acad. Sci. U. S. A. 2015, 112, 2658-2663.

(20) Cao, Y.; Dong, S.; Liu, S.; He, L.; Gan, L.; Yu, X.; Steigerwald, M. L.; Wu, X.; Liu, Z.; Guo, X. Angew. Chem., Int. Ed. 2012, 51, $12228-12232$.

(21) Wood, J. D.; Doidge, G. P.; Carrion, E. A.; Koepke, J. C.; Kaitz, J. A.; Datye, I.; Behnam, A.; Hewaparakrama, J.; Aruin, B.; Chen, Y.; Dong, H.; Haasch, R. T.; Lyding, J. W.; Pop, E. Nanotechnology 2015, 26, 055302 .

(22) Ferrari, A. C.; Meyer, J. C.; Scardaci, V.; Casiraghi, C.; Lazzeri, M.; Mauri, F.; Piscanec, S.; Jiang, D.; Novoselov, K. S.; Roth, S.; Geim, a. K. Phys. Rev. Lett. 2006, 97, 187401.

(23) Casiraghi, C.; Hartschuh, A.; Qian, H.; Piscanec, S.; Georgi, C.; Fasoli, A.; Novoselov, K. S.; Basko, D. M.; Ferrari, A. C. Nano Lett. 2009, 9, 1433-1441.

(24) Ferrari, A. C.; Basko, D. M. Nat. Nanotechnol. 2013, 8, 235-246.

(25) Li, X.; Cai, W.; An, J.; Kim, S.; Nah, J.; Yang, D.; Piner, R; Velamakanni, A.; Jung, I.; Tutuc, E.; Banerjee, S. K.; Colombo, L.; Ruoff, R. S. Science 2009, 324, 1312-1314.

(26) Simmons, J. G. J. Appl. Phys. 1963, 34, 1793-1803.

(27) Oshima, C.; Nagashima, A. J. Phys.: Condens. Matter 1997, 9, 120

(28) Yuan, H.; Chang, S.; Bargatin, I.; Wang, N. C.; Riley, D. C.; Wang, H.; Schwede, J. W.; Provine, J.; Pop, E.; Shen, Z. X.; Pianetta, P. A.; Melosh, N. A.; Howe, R. T. Nano Lett. 2015, 15, 6475-6480. 
(29) Song, S. M.; Park, J. K.; Sul, O. J.; Cho, B. J. Nano Lett. 2012, 12, 3887-3892.

(30) Shi, Y.; Kim, K. K.; Reina, A.; Hofmann, M.; Li, L.-J.; Kong, J. ACS Nano 2010, 4, 2689-2694.

(31) Kvashnin, D. G.; Sorokin, P. B.; Brüning, J. W.; Chernozatonskii, L. A. Appl. Phys. Lett. 2013, 102, 183112.

(32) Cretu, O.; Botello-Mendez, A. R.; Janowska, I.; Pham-Huu, C.; Charlier, J. C.; Banhart, F. Nano Lett. 2013, 13, 3487-3493.

(33) Jin, C.; Lan, H.; Peng, L.; Suenaga, K.; Iijima, S. Phys. Rev. Lett. 2009, 102, 205501.

(34) Chuvilin, A.; Meyer, J. C.; Algara-Siller, G.; Kaiser, U. New J. Phys. 2009, 11, 083019.

(35) Erdogan, E.; Popov, I.; Rocha, C. G.; Cuniberti, G.; Roche, S.; Seifert, G. Phys. Rev. B: Condens. Matter Mater. Phys. 2011, 83, 41401. (36) Zhang, G. P.; Fang, X. W.; Yao, Y. X.; Wang, C. Z.; Ding, Z. J.; Ho, K. M. J. Phys.: Condens. Matter 2011, 23, 025302.

(37) Topsakal, M.; Ciraci, S. Phys. Rev. B: Condens. Matter Mater. Phys. 2010, 81, 24107.

(38) Postma, H. W. C. Nano Lett. 2010, 10, 420-425. 